\title{
Microsecond time resolved X-ray diffraction for the fast determination of fatigue behavior beyond one billion cycles
}

\author{
D. Vinci, V. Jacquemain, C. Cheuleu, V. Michel, O. Castelnau, V. Favier, N. Ranc \\ Laboratoire PIMM, Arts et Métiers Institute of Technology, CNRS, HESAM Université, 151 Boulevard de l'Hôpital, 75013 Paris, \\ France
}

doriana.vinci@ensam.eu

\begin{abstract}
Many mechanical structures are submitted to repeated loadings during their life span and can break under stress lower than the ultimate tensile stress. This phenomenon, called fatigue of materials, has attracted the scientific community attention due to its effect in many industrial sectors, such as the transport, aeronautic and energy. Fatigue design is thus crucial in engineering and it requires the accurate characterization of material behavior under cyclic loadings to ensure the safety and reliability of structures throughout their life. It is presently common to find mechanical systems subjected to several billion cycles, in what is called the gigacycle fatigue domain or very high cycle fatigue (VHCF) domain [1]. The characterization of the fatigue behavior of materials have been largely investigated with fatigue tests requiring long testing time with standard laboratory. To overcome this inconvenient new approaches using ultrasonic fatigue machines have been developed during the last decades. In particular, the present research group developed recently a new method for the fast determination of fatigue behavior interpreting diffraction patterns with a temporal resolution of $\sim 1 \mu$ s during an ultrasonic fatigue test and loading frequency of about $20 \mathrm{kHz}$.

The present study points on the estimation of the amount of energy stored by the specimen during its deformation due to an ultrasonic fatigue loading. This energy is a crucial parameter as it is strictly related to the fatigue damage and can be estimated from the intrinsic dissipation and the mechanical work supplied to the specimen.

X-ray diffraction analysis were performed to measure the supplied work by integrating over one fatigue cycle of the product of the strain rate by the stress. In particular, pure copper and steel specimens were loaded using a $20 \mathrm{kHz}$ ultrasonic fatigue machine mounted on the six-circle diffractometer available at the DiffAbs beamline on the SOLEIL synchrotron facility in France. Then, in order to obtain the mechanical work: 1) from the shift of Bragg peaks is possible to estimate the total stress applied to the sample, 2) from both the broadening and shift of peaks one can measure the mean elastic lattice strain distribution, and 3) from the peak broadening the fluctuation of elastic strain is deduced, providing information about intragranular strain heterogeneity and dislocation density.
\end{abstract}

[1] Bathias, C. \& Paris, P. (2005). Gigacycle Fatigue in Mechanical Practice. New York: Marcel Dekker.

Keywords: ultrasonic fatigue tests; very high cycle fatigue; $X$-ray diffraction; time-resolved stress measurement 